\title{
Pratiques
}

Linguistique, littérature, didactique

183-184|2019

oralité, littératie

\section{Vocalisation, voix}

\section{Kathie Birat}

\section{OpenEdition}

Journals

Édition électronique

URL : http://journals.openedition.org/pratiques/6822

DOI : $10.4000 /$ pratiques. 6822

ISSN : 2425-2042

\section{Éditeur}

Centre de recherche sur les médiations (CREM)

\section{Référence électronique}

Kathie Birat, « Vocalisation, voix », Pratiques [En ligne], 183-184 | 2019, mis en ligne le 30 décembre

2019, consulté le 09 octobre 2020. URL : http://journals.openedition.org/pratiques/6822 ; DOI :

https://doi.org/10.4000/pratiques.6822

Ce document a été généré automatiquement le 9 octobre 2020.

(c) Tous droits réservés 


\title{
Vocalisation, voix
}

\author{
Kathie Birat
}

1 La vocalisation se réfère à la production du son par la voix. Le Dictionnaire de linguistique et des sciences du langage (Dubois et al., 1994, p. 508) spécifie que «le terme de vocalisation est parfois employé [...] comme synonyme de voisement ou sonorisation pour désigner la mise en vibration des cordes vocales qui produit la voix ». Comme le suggère cette définition, la vocalisation est inséparable de la voix. Le Dictionnaire cité cidessus définit la voix comme «l'ensemble des ondes sonores produites dans le larynx par la vibration des cordes vocales sous la pression de l'air subglottique » (ibid., p. 509).

2 La vocalisation est le plus souvent définie en termes linguistiques, bien que cette approche révèle rapidement ses limites. D. Crystal (1980, p. 374, ma traduction) la définit comme "un terme général utilisé en linguistique et en phonétique pour désigner une énonciation vue uniquement comme une suite de sons sans prise en compte de sa structure linguistique ». La vocalisation n'est pourtant pas une simple actualisation du potentiel de l'appareil phonatoire. Comme le souligne C. Détrie (et al., 2017), l'aspect « matériel et concret de la parole » représenté par les sons et les « bruits extralinguistiques » constitue un élément essentiel de "l'analyse des discours oraux » par les indices qu'il livre « sur le positionnement subjectif du locuteur».

En outre, elle n'échappe pas à la relation paradoxale entre son et sens mise en évidence par M. Dolar dans Une voix et rien d'autre. Si, comme dit M. Dolar, la voix « fonctionne comme porteuse d'un énoncé, comme support d'un mot, d'une phrase, d'un discours » toute tentative pour établir une « linguistique de la voix » bute sur la nécessité de vider la voix de sa substance sonore pour la faire entrer dans le système différentiel de la phonologie. Dans sa discussion des "usages présymboliques de la voix ", ceux qu'il appelle «les voix physiologiques", «le babil» et le «cri», M. Dolar explique : «il semble que nous ayons affaire à une voix externe à la structure, et pourtant, cette extériorité apparente atteint le cœur même de la structure: elle symbolise le geste signifiant précisément en ne signifiant rien en particulier [...]» (Dolar, 2012, p. 39). Pour exprimer le statut paradoxal de la voix, cette impossibilité à séparer « la voix "en chair et en os"(comme Jakobson le dira quelques décennies plus tard) » de cette « entité sans chair ni os purement définie par sa fonction » (ibid., p. 25), il caractérise la voix 
métaphoriquement comme "un angle mort de la production de signification ». (ibid., p. 24)

4 Cette difficulté à séparer voix et vocalisation se retrouve chez d'autres auteurs préoccupés par la voix, la vocalisation et l'oralité. Paul Zumthor (1982, p. 233) s'étonne que « parmi toutes nos disciplines instituées, nous n'ayons pas encore une science de la voix ». S'il constate que " la voix est une chose : on en décrit les qualités matérielles, le ton, le timbre, l'ampleur, la hauteur, le registre... » il reconnaît en même temps qu'« à chacune d'elles la coutume attache une valeur symbolique.» (ibid.) Il définit la parole comme "le langage vocalisé, phoniquement réalisé dans l'émission de la voix », mais résume à sa façon le paradoxe identifié par $\mathrm{M}$. Dolar en désignant la voix comme "parole sans paroles" (Dolar, 2012, p. 234). Comme M. Dolar il voit le rapport entre voix et verbe - «ce que les premiers théologiens du langage, au XVI ${ }^{\mathrm{e}}$ siècle nommèrent le verbe [...] », la voix « que viennent habiter les mots » (ibid., p. 234-235), rapport dont parlera M. Dolar en évoquant "la voix comme condition de la révélation du Verbe " (ibid., p. 24). La glossolalie, "ce phénomène verbo-moteur qui apparaît souvent lors d'extases religieuses et qui consiste dans l'émission non contrôlée de phonèmes pseudolangagiers ", une forme de vocalisation présente dans de nombreuses cultures ", démontre bien cette tendance à associer la vocalisation à la spiritualité. (Denis,1989, p. 104). La vocalisation, en dépit de son ancrage dans un phénomène physique, possède une dimension culturelle qu'H. Meschonnic explique, après M. Mauss, par le fait que «le corps est historique, culturel.» (Meschonnic, 1993, p. 88). À l'instar de M. Dolar, Meschonnic met en évidence l'impossibilité de réduire la voix au "phonique » dans le sens purement physique du terme, «car l'énergie qui la produit engage aussi le corps vivant avec son histoire». (ibid., p. 87.)

5 Cette dimension culturelle de la vocalisation joue un rôle dans l'oralité. Pour Meschonnic, c'est la notion de rythme qui permet de sortir du «dualisme anthropologique " lié au signe Saussurien et de cesser de confondre oralité et parlé: "L'idée semble pourtant d'une évidence ancienne, incontestable, que l'oral soit le parlé, que le passage à l'écrit soit la perte de la voix, du geste, de la mimique, de tout l'accompagnement du corps à l'énoncé proféré. » (Meschonnic, 1982, p. 16) La vision binaire $d u$ rapport entre écriture et oralité serait, d'après Meschonnic, l'héritage «d'une vision ethnologique de l'oralité, identifiée au parlé.» (16) Elle s'appuie sur une réduction de la vocalisation à son rôle de support de l'énoncé dans la langue parlée, entraînant une vision simpliste du rapport écriture/oralité à l'image des autres dualités introduites par le primat du signe en linguistique saussurienne. Meschonnic fait appel au rythme, comme M. Dolar fait appel à la voix, pour prendre « en compte des modes de signifier qui débordent du signe »(Meschonnic, 1982, p. 8).

6 Les travaux de W. Ong sur l'oralité reposent sur une approche similaire de la vocalisation et de son rôle dans la production du sens en contexte d'oralité. Comme le montre Ong, l'étude de la composition de la poésie homérique par Milton Parry a permis d'approfondir la compréhension de l'impact de la composition orale sur la forme de la poésie aussi bien que sur sa manière de signifier. D'un point de vue historique, Marcel Jousse a "montré le lien entre les schémas rythmiques oraux, la respiration, les gestes et la symétrie bilatérale du corps dans les targums de l'époque araméenne et hellénique, et donc aussi en hébreu ancien ». (Ong, 2014 [1982], p. 54). Ong affirme qu'avec l'introduction de l'écriture, la composition reste marquée par un contexte d'oralité: "L'esprit n'a initialement aucune véritable ressource 
chirographique. Vous griffonnez sur un support des mots que vous imaginez prononcer à voix haute dans un contexte oral plausible. » (ibid., p. 46-47). En régime d'oralité dite «primaire », la vocalisation, réelle ou potentielle, conditionne la production aussi bien que la réception des discours. Elle n'intervient pas uniquement lors de la performance, mais joue un rôle structurant dans la vie sociale. D'après W. Ong (ibid., p.54) «la construction d'une pensée élaborée dans une culture orale est liée à la communication». La vocalisation dans un contexte d'oralité met souvent en jeu le corps dans sa totalité : "Comme nous l'avons vu, au contraire de la parole écrite, le monde oral n'existe jamais dans un contexte exclusivement verbal. Les mots parlés sont toujours des modifications d'une situation totale et existentielle, qui implique invariablement le corps. Dans la communication orale, l'activité physique au-delà de la simple mise en voix n'est ni fortuite ni forcée mais naturelle et même inévitable.» (ibid., p. 87).

7 Cette vision de la voix et de la vocalisation dans leur rapport avec l'oralité trouve un appui théorique dans les travaux de P. Koch et W. Oesterreicher (2001). En établissant une distinction entre l'aspect médial et l'aspect conceptionnel d'un énoncé, ils permettent de ne pas confondre la "réalisation phonique " d'un énoncé avec son «aspect conceptionnel». Alors que «la différence entre les codes phonique et graphique [qui concerne l'aspect médial] représente une dichotomie au sens strict", « langage parlé et langage écrit [qui relèvent de l'aspect conceptionnel] correspondent aux deux extrêmes d'un continuum communicatif» (Koch \& Oesterreicher, 2001, p. 585). La vocalisation dans sa dimension physique relève de l'aspect médial et permet de distinguer l'oral de l'écrit. L'oralité, par contre, dans une perspective qui répond aux soucis de Meschonnic et d'Ong, peut être envisagée d'après le continuum communicatif qui permet de prendre en compte la possibilité d'une «distance communicative » qui n'est pas incompatible avec une réalisation phonique: "Mais l'absence du code graphique n'implique nullement l'absence de la distance communicative, même dans les cultures dites "orales", qui connaissent l'immédiat phonique [...] aussi bien que certaines formes-bien particulières il est vrai-de la distance phonique [...]. Ces manifestations d'une distance poétique ou rituelle sans écriture constituent ce que l'on pourrait appeler "l'oralité élaborée" ». (ibid., 587)

Les notions de " voix » et de "vocalisation » restent intimement liées. Toute tentative pour établir une distinction qui accorderait un statut métaphorique à la première en cantonnant la deuxième au corps, perçu dans sa dimension purement matérielle, rencontre les paradoxes révélés par les pratiques orales.

\section{BIBLIOGRAPHIE}

CRYSTAL, D. (1980). A Dictionary of Linguistics and Phonetics. Londres : A. Deutsch.

DENIS, J.-P., (1989). « Glossolalies : vestiges d'une oralité première », Études littéraires 22 (2), p. 99-108. 
DÉTRIE, C., et al.. (2017) [2001]. Termes et concepts pour l'analyse du discours. Paris : Honoré Champion. DOLAR, M. (2012). Une voix et rien d'autre. Trad. de l'anglais par C. Vivier. Paris : Éditions Nous. DUBoIs, J. et al. (1994) Dictionnaire de linguistique et des sciences du langage. Paris : Larousse, . KOCH, P. \& OESTERREICHER, W. (2001) : « Gesprochene Sprache und geschriebene Sprache ». In : Holtus, G., Metzeltin, M., \& Schmitt, C. (éds), Lexikon der romanistischen Linguistik. Band I, 2: Methodologie. Tubingue : Max Niemeyer Verlag, p. 584-627.

MESCHONNIC, H. (1982). « Qu'entendez-vous par oralité ? », Langue francaise 56, p. 6-23.

MEschonNIC, H. (1993). «L'Oralité, poétique de la voix ». In : Revel, N. \& Rey-Hulman, D. (éds). Pour une anthologie des voix. Paris : l'Harmattan, p. 83-107.

ONG, W. J. (2014) [1982]. Oralité et écriture : la technologie de la parole. Trad. de l'anglais par H. Hiessler. Paris : Les Belles Lettres.

ZUMTHOR, P. (1982). «Considérations sur les valeurs de la voix », Cahiers de civilisation médiévale 99-100, p. 233-238.

\section{AUTEUR}

\section{KATHIE BIRAT}

Université de Lorraine, F-57000 Metz, France 